\title{
ON FINITELY GENERATED AND PROJECTIVE EXTENSIONS OF BANACH ALGEBRAS
}

\author{
JOAN VERDERA
}

\begin{abstract}
We show that a finitely generated projective extension $B$ of a commutative complex unitary Banach algebra $A$ induces an open mapping $\pi$ between the carrier spaces. We next prove that if $\pi$ is a local homeomorphism then $B$ contains an inertial subalgebra. Finally we present a necessary and sufficient condition for $B$ to be uniform if $A$ is.
\end{abstract}

1. Introduction. Throughout this paper Banach algebra means a commutative Banach algebra over the complex field $\mathrm{C}$ with an identity element 1 . If $A$ is a Banach algebra, then its carrier space, endowed with the Gelfand topology, is denoted by $M(A)$ and its Shilov boundary by $\partial(A)$. For each $a \in A$ we denote by $\hat{a}$ the Gelfand transform of $a$. The Jacobson radical of $A$ is written $R(A)$.

From now on $B$ will denote a finitely generated projective extension of a fixed Banach algebra $A$. We suppose $B$ to be endowed with Magid's norm under which $B$ is also a Banach algebra [10, Theorem 4, p. 138]. We call $\pi$ the projection of $M(B)$ onto $M(A)$ induced by the inclusion of $A$ into $B$. The above situation is illustrated by the following example: take $B=A[x] /(\alpha(x))$ where $\alpha(x)$ is a monic polynomial in $A[x]$ (the so-called Arens-Hoffman extensions of $A$ ). For such extensions, the structure of the projection $\pi$ as well as the study of the properties that $B$ can inherit from $A$ have been the main subject in a series of papers ([4], [7], [8]). The purpose of this paper is to establish some results along these lines for the more general situation.

To state the main results it is convenient to introduce a definition.

If $\psi \in M(B), \phi=\pi(\psi)$, and $m_{\phi}=\operatorname{Ker} \phi$, then $B / m_{\phi} B$ is a finite dimensional C-algebra whose carrier space is naturally homeomorphic to $\pi^{-1}(\phi)$. Then we define the multiplicity $m(\psi)$ of $\psi$ as the complex dimension of $e\left(B / m_{\phi} B\right)$, where $e$ is the idempotent element of $B / m_{\phi} B$ such that the support of $\hat{e}$ is $\{\psi\}$.

THEOREM 1. If $\psi_{1} \in M(B)$ and $\pi^{-1}\left(\pi\left(\psi_{1}\right)\right)=\left\{\psi_{1}, \ldots, \psi_{m}\right\}$, then given any neighborhood $W$ of $\psi_{1}$, there exist mutually disjoint neighborhoods $V_{i}$ of $\psi_{i}$ and a neighborhood $U$ of $\pi\left(\psi_{1}\right)$ such that:

(a) $\pi\left(V_{i}\right)=U$ for all $i$

(b) $\pi^{-1}(U)=\cup_{i=1}^{m} V_{i}$,

(c) $V_{1} \subset W$,

Received by the editors November 29, 1978 and, in revised form, September 19, 1979 and January 30, 1980.

AMS (MOS) subject classifications (1970). Primary 46H99; Secondary 13B20.

Key words and phrases. Banach algebra, finitely generated projective extension, inertial subalgebra. 
(d) $m\left(\psi_{i}\right)=\Sigma_{\theta \in \pi^{-1}(\phi) \cap v_{i}} m(\theta), \phi \in U, i=1, \ldots, m$.

In particular $\pi$ is open.

THEOREM 2. If $\pi$ is a local homeomorphism, then $B$ contains an $A$-separable subalgebra $B_{0}$ such that $B=B_{0}+R(B)$.

TheOREM 3. Suppose $A$ is a uniform algebra. Then the following are equivalent:

(a) $B$ is a uniform algebra.

(b) $m(\psi)=1$ for each $\psi \in \partial(B)$.

Theorem 1 and Theorem 3 generalize analogous results for the Arens-Hoffman case in [7] and [4], respectively. The subalgebras whose existence is established in Theorem 2 are usually called inertial subalgebras. Other existence theorems for inertial subalgebras have been found by Ingraham in [6] in a more general context.

In the following sections we proceed to prove the theorems stated above. For basic facts and terminology concerning algebras over commutative rings, (algebraically) separable algebras and projective modules the reader is referred to [3].

2. Proof of Theorem 1. ${ }^{1}$ If $\alpha(x)=\sum_{i=0}^{n} \alpha_{i} x^{i} \in A[x]$ and $\phi \in M(A)$ we set $\alpha_{\phi}(x)=\sum_{i=0}^{n} \phi\left(\alpha_{i}\right) x^{i}$ and $Z\left(\alpha_{\phi}\right)=\left\{\lambda \in C / \alpha_{\phi}(\lambda)=0\right\}$. For $\lambda \in Z\left(\alpha_{\phi}\right)$, we let $M\left(\lambda, \alpha_{\phi}\right)$ stand for the multiplicity of $\lambda$ as a root of $\alpha_{\phi}$. If $\alpha(x)$ is monic we write $A_{\alpha}$ for the Arens-Hoffman extension $A[x] /(\alpha(x))$ and $\pi_{\alpha}$ for the projection of $M\left(A_{\alpha}\right)$ onto $M(A)$. We recall that a finitely generated projective module $M$ is said to have a well-defined rank $n$ if for any prime ideal $p$ of $A$ the localized module $M_{p}$ (which is free) has rank $n$ over the local ring $A_{p}$. In this case, we have $\operatorname{dim}_{\mathrm{C}} M / m_{\phi} M=n$ for each $\phi \in M(A)$.

LEMMA. Suppose that $B$ has well defined rank, say $n$. Then for each $b \in B$ there is a monic polynomial $\alpha(x) \in A[x]$, of degree $n$, and a continuous mapping $f^{*}: M(B) \rightarrow$ $M\left(A_{\alpha}\right)$, commuting with projections, such that:

(a) $f^{*}$ is onto.

(b) $M\left(\psi(b), \alpha_{\pi(\psi)}\right)=\Sigma m(\theta), \psi \in M(B)$,

the sum being taken over those $\theta \in M(B)$ satisfying $f^{*}(\theta)=f^{*}(\psi)$.

Proof. Fixed $b \in B$, we consider the $A$-endomorphism $u$ of $B$ defined by $u(c)=c b, c \in B$. Let $\alpha(x)=\operatorname{det}(x I-u)$ be the characteristic polynomial of $u$ (see [1, exercise 9 (c), p. 148] for a definition). We have $\alpha(b)=0$ and $n=$ degree $\alpha(x)$. Let $f$ be the $A$-algebra homomorphism from $A_{\alpha}$ into $B$ determined by the condition $f([x])=b$, where $[x]$ denotes the class of $x$ in $A_{\alpha}$. Let $f^{*}$ be the dual map of $f$. In order to prove (a) we note that a character in $M\left(A_{\alpha}\right)$ is given by a pair $(\phi, \lambda)$ with $\phi \in M(A)$ and $\lambda \in Z\left(\alpha_{\phi}\right)$, acting in the following way

$$
\sum_{i=0}^{n-1} a_{i}[x]^{i} \rightarrow \sum_{i=0}^{n-1} \phi\left(a_{i}\right) \lambda^{i}, \quad a_{i} \in A \text { for each } i .
$$

Thus it follows that $f^{*}(\psi)=(\pi(\psi), \psi(b))$.

\footnotetext{
${ }^{1}$ This proof is not the original proposed by the author, which was longer. I am indebted to the referee for the present proof as well as for the statement he suggested of the lemma in this section.
} 
We write $u_{\phi}$ for the C-endomorphism of $B / m_{\phi} B$ obtained from $u$ by factoring by the ideal $m_{\phi} B$. For each $\psi_{i} \in \pi^{-1}(\phi)$, let $e_{i}$ be the idempotent of $B / m_{\phi} B$ such that the support of $\hat{e}_{i}$ is $\left\{\psi_{i}\right\}, 1 \leqslant i \leqslant \operatorname{card} \pi^{-1}(\phi)$. Then $B / m_{\phi} B$ is the direct sum of the subalgebras $e_{i}\left(B / m_{\phi} B\right)$, which are invariant under $u_{\phi}$. It is easily seen that the unique eigenvalue of the restriction of $u_{\phi}$ to $e_{i}\left(B / m_{\phi} B\right)$ is $\psi_{i}(b)$, and thus

$$
\alpha_{\phi}(x)=\prod_{i}\left(x-\psi_{i}(b)\right)^{m\left(\psi_{i}\right)}, \quad \phi \in M(A) .
$$

Since the character in $M\left(A_{\alpha}\right)$ determined by the pair $(\phi, \lambda)$ is in $\operatorname{Im} f^{*}$ if and only if $\lambda=\psi_{i}(b)$ for some $\psi_{i} \in \pi^{-1}(\phi),(1)$ proves (a).

To prove (b) it is enough to observe that for $\psi, \theta \in M(B), f^{*}(\psi)=f^{*}(\theta)$ is equivalent to $\psi(b)=\theta(b)$ and $\pi(\psi)=\pi(\theta)$.

Now we can proceed to prove Theorem 1 . By $[3,4.11$, p. 31], there are mutually orthogonal idempotents $e_{1}, \ldots, e_{p}$ of $A$, such that $e_{1}+\cdots+e_{p}=1$ and $e_{i} B$ is a finitely generated projective extension of $e_{i} A$ with a well defined rank (over $e_{i} A$ ) for each $i$. Hence without loss of generality we may assume that $B$ has a well-defined rank over $A$, say $n$.

If we put $B_{0}=B \otimes_{A} C(M(A)$ ) (we denote by $C(X)$ the algebra of continuous complex functions over a compact space $X$ ), then $B_{0}$ is a finitely generated projective extension of $C(M(A))$ [3, 2.1, p. 12] with a well-defined rank over $C\left(M(A)\right.$ ) (in fact, the rank is $n$ ). It can easily be seen that $M\left(B_{0}\right)$ is homeomorphic to $M(B)$ under the map $h$ induced by the $A$-algebra homomorphism $b \rightarrow b \otimes 1$ of $B$ into $B_{0}$. But $h$ preserves the multiplicity, so we may also assume that $A=$ $C(M(A))$.

Let $b_{1}, \ldots, b_{k}$ be a set of generators of $B$ over $A$ and let $\alpha_{1}, \ldots, \alpha_{k}$ be monic polynomials in $A[x]$ satisfied by $b_{1}, \ldots, b_{k}$ respectively. Put $B_{1}=$ $\left(\left(\cdots\left(A_{\alpha_{1}}\right)_{\alpha_{2}}\right) \cdots\right)_{\alpha_{k}}$, the iterated Arens-Hoffman extension generated by $\alpha_{1}, \ldots, \alpha_{k}$. By construction we have a continuous $A$-algebra homomorphism from $B_{1}$ onto $B$. Applying $k$ times $\left[8,4.2\right.$, p. 564] we obtain that $\hat{B}_{1}$ is uniformly dense in $C\left(M\left(B_{1}\right)\right)$. From this it follows that $\hat{B}$ is uniformly dense in $C(M(B))$.

Using this fact, we can consider $b \in B$ and $\varepsilon>0$ such that $\hat{b}$ separates the points of $\pi^{-1}\left(\pi\left(\psi_{1}\right)\right)$ and

$$
V\left(\psi_{1} ; b, \varepsilon\right)=\left\{\theta \in M(B) /\left|\theta(b)-\psi_{1}(b)\right|<\varepsilon\right\} \subset W,
$$

where the equality is a definition. Let $\alpha(x)$ and $f^{*}$ be related to $b$ as in the lemma. Then $V\left(\psi_{1} ; b, \varepsilon\right)=f^{*-1}\left(V\left(f^{*}\left(\psi_{1}\right) ;[x], \varepsilon\right)\right)$. By Propositions 1.1 and 1.3 of [7], there are mutually disjoint $M$-neighborhoods $V_{1}^{*}, \ldots, V_{m}^{*}$ of the points $f^{*}\left(\psi_{1}\right), \ldots, f^{*}\left(\psi_{m}\right)$ respectively, such that $\pi_{\alpha}\left(V_{i}^{*}\right)=\pi_{\alpha}\left(V_{1}^{*}\right)$ for each $i, \pi_{\alpha}^{-1}\left(\pi_{\alpha}\left(V_{1}^{*}\right)\right)$ $=\cup_{i} V_{i}^{*}$ and $V_{1}^{*} \subset V\left(f^{*}\left(\psi_{1}\right) ;[x], \varepsilon\right)$. The sets $U=\pi_{\alpha}\left(V_{1}^{*}\right), V_{i}=f^{*-1}\left(V_{i}^{*}\right)$ satisfy (a), (b) and (c). ( $U$ is a neighborhood of $\pi\left(\psi_{1}\right)$ because $\pi_{\alpha}$ is open owing to [7, 1.2, p. 358]). In order to prove (d) fix $\phi \in U$ and $i \in\{1, \ldots, m\}$. Since $\hat{b}$ separates the points of $\pi^{-1}\left(\pi\left(\psi_{1}\right)\right)$ and because of the definition of $M$-neighborhood we have

$$
m\left(\psi_{i}\right)=M\left(\psi_{i}(b), \alpha_{n\left(\psi_{1}\right)}\right)=\sum_{\lambda} M\left(\lambda, \alpha_{\phi}\right)
$$

where $\lambda$ runs over the set $\left\{\theta(b) / \theta \in \pi^{-1}(\phi) \cap V_{i}\right\}$. 
On the other hand, by part (b) of the lemma we have

$$
M\left(\lambda, \alpha_{\phi}\right)=\sum_{\eta} m(\eta), \text { for each } \lambda,
$$

where $\eta$ runs over the set $\left\{\theta \in \pi^{-1}(\phi) / \theta(b)=\lambda\right\}$. Now part (d) of the theorem follows from (2) and (3). Theorem 1 is thereby proved.

Using Theorem 1 and $[9,1.6$, p. 677] we immediately obtain the following.

Corollary. $\delta(B)=\pi^{-1}(\delta(A))$.

Remark. A point $\psi \in M(B)$ is called a singular point if $\pi$ fails to be a local homeomorphism at $\psi$. From the (topological) conditions (a), (b) and (c) of the theorem and the uniform boundedness of the cardinal of the fibers of $\pi$, it easily follows that the (closed) set of all singular points of $M(B)$, as well as its projection, has empty interior.

3. Proof of Theorem 2. We need a lemma.

LEMMA. If $\pi$ is a homeomorphism, then $\hat{B}=\hat{A}$.

Proof. The same argument used at the beginning of the proof of Theorem 1 shows that we may suppose that $B$ has a well-defined rank, say $n$. For $b \in B$ let $\alpha(x)=\alpha_{0}+\alpha_{1} x+\cdots+\alpha_{n-1} x^{n-1}+x^{n}$ be as in the lemma preceding Theorem 1. From (1) we obtain $\hat{b}=\left(-n^{-1} \alpha_{n-1}\right)^{\wedge} \in A$. This is the proof.

Now we can give a proof of Theorem 2 . First recall that by a strongly separable extension is meant a finitely generated projective separable extension. It is well known that $B \otimes_{A} B$ is a finitely generated projective extension of $A$. Also $M\left(B \otimes_{A} B\right)$ is (homeomorphic to)

$$
M(B) \times_{M(A)} M(B)=\{(\psi, \theta) \in M(B) \times M(B) / \pi(\psi)=\pi(\theta)\}
$$

(cf. [10, Lemma 6, p. 139]). But $M(B) \times_{M(A)} M(B)$ is a finite covering space of $M(A)$ because $M(B)$ is. Then, $C\left(M(B) \times_{M(A)} M(B)\right)$ and $C(M(B))$ are strongly separable extensions of $C(M(A))$ [2, Theorem 2, p. 30]. On the other hand $C(M(B)) \otimes_{C(M(A))} C(M(B))$ is also a strongly separable extension of $C(M(A))[3$, 1.6, p. 43]. Thus, Theorem 2 of [2] allows us to identify, via the Gelfand transform, $C(M(B)) \otimes_{C(M(A))} C(M(B))$ with $C\left(M(B) \times_{M(A)} M(B)\right)$.

If $e_{0}$ is the separability idempotent for $C(M(B))$ [3, p. 40], by the Shilov idempotent theorem there is an idempotent $e \in B \otimes_{A} B$ such that $\hat{e}=e_{0}$. Set $B_{0}=\{b \in B /(b \otimes 1-1 \otimes b) e=0\}$. If $e$ is written as $e=\Sigma_{k} x_{k} \otimes y_{k}, x_{k}, y_{k} \in$ $B$, we show that the following relations hold:

$$
\begin{aligned}
\sum_{k, j} x_{k} \otimes x_{j} \otimes y_{k} y_{j} & =\sum_{k, j} x_{k} \otimes x_{j} y_{k} \otimes y_{j}, \\
\sum_{k} x_{k} \otimes y_{k} & =\sum_{k} y_{k} \otimes x_{k}, \\
\sum_{k} x_{k} y_{k} & =1 .
\end{aligned}
$$


To prove them, consider the $A$-algebra homomorphisms

$$
\begin{array}{ll}
\varphi_{1}: B \otimes_{A} B \rightarrow B \otimes_{A} B \otimes_{A} B, & \varphi_{1}(x \otimes y)=x \otimes(1 \otimes y) e, \\
\varphi_{2}: B \otimes_{A} B \rightarrow B \otimes_{A} B \otimes_{A} B, & \varphi_{2}(x \otimes y)=x \otimes(y \otimes 1) e .
\end{array}
$$

Put $u_{i}=\varphi_{i}(e), i=1,2$. By the definition of separability idempotent we have

$$
\hat{u}_{1}=\sum_{k} \hat{x}_{k} \otimes\left(1 \otimes \hat{y}_{k}\right) e_{0}=\sum_{k} \hat{x}_{k} \otimes\left(\hat{y}_{k} \otimes 1\right) e_{0}=\hat{u}_{2}
$$

and since $u_{1}$ and $u_{2}$ are idempotents we obtain $u_{1}=u_{2}$, which is (4).

(5) follows from the relation $\Sigma_{k} \hat{x}_{k} \otimes \hat{y}_{k}=\Sigma_{k} \hat{y}_{k} \otimes \hat{x}_{k}$ which is obtained by appealing to the uniqueness, in the commutative case, of separability idempotents.

Now consider the commutative diagram shown below, where the vertical arrows are the Gelfand transforms and the horizontal ones are defined by $x \otimes y \rightarrow x y$

$$
\begin{array}{ccc}
B \otimes_{A} B & \stackrel{\mu}{\rightarrow} & B \\
\downarrow & & \downarrow \\
C(M(B)) \otimes_{C(M(A))} C(M(B)) & \rightarrow & C(M(B))
\end{array}
$$

Since $e_{0}$ is the separability idempotent of $C(M(B))$ we have $\widehat{\mu(e)}=1$. But $\mu(e)$ is idempotent, so $\mu(e)=1$. This proves (6).

Next we will show that $e \in B_{0} \otimes_{A} B_{0}$ by adapting an argument of Magid [11, Theorem 1.3, p. 91]. Let $\varphi$ be the $A$-homomorphism of $B$ into $B \otimes_{A} B, \varphi(b)=$ $(b \otimes 1-1 \otimes b) e, b \in B$; thus the sequence

$$
(0) \rightarrow B_{0} \hookrightarrow B \stackrel{\varphi}{\rightarrow} B \otimes_{A} B
$$

is exact. Tensoring with $B$ over $A$ we obtain the exact sequence (recall that $B$ is $A$-flat)

$$
(0) \rightarrow B \otimes_{A} B_{0} \rightarrow B \otimes_{A} B \stackrel{1 \otimes_{\varphi}}{\rightarrow} B \otimes_{A} B \otimes_{A} B .
$$

By $(3)(1 \otimes \varphi)(e)=0$; then $e \in B \otimes_{A} B_{0}$ and so we can assume that each $y_{k}$ is in $B_{0}$. We set $\mu_{0}=\mu / B \otimes B_{0}$ and $g(b)=(b \otimes 1) e, b \in B$, so that $g$ is a $B_{0}$-homomorphism of $B$ into $B \otimes_{A} B_{0}$. We have $\mu_{0} \circ g=I_{B}$ (by (6)), which implies that $B$ is a $B_{0}$-factor of $B \otimes_{A} B_{0}$ and hence $B_{0}$-projective. Using [1, Exercise 4, p. 147], we conclude that $B_{0}$ is a $B_{0}$-factor of $B$, hence an $A$-factor of $B$. Therefore $B_{0}$ is $A$-projective and so $A$-flat.

Tensoring (7) with $B_{0}$ we obtain the exact sequence

$$
(0) \rightarrow B_{0} \otimes_{A} B_{0} \rightarrow B_{0} \otimes_{A} B \stackrel{1 \otimes \varphi}{\rightarrow} B_{0} \otimes_{A} B \otimes_{A} B \text {. }
$$

By (5) $e \in B_{0} \otimes_{A} B$ and by (4) $(1 \otimes \varphi)(e)=0$, so $e \in B_{0} \otimes_{A} B_{0}$.

We claim now that $\hat{B}_{0}$ separates the points of $M(B)$. Let $\psi, \theta \in M(B)$ with $\psi \neq \theta$ and $\pi(\psi)=\pi(\theta)$. Suppose that $\psi\left(y_{k}\right)=\theta\left(y_{k}\right)$ for each $k$. Using the fact that $e_{0}$ is the characteristic function of the set $\left\{\left(\psi^{\prime}, \theta^{\prime}\right) \in M(B) \times_{M(A)} M(B) / \psi^{\prime}=\theta^{\prime}\right\}$, we reach a contradiction, namely

$$
0=e_{0}(\psi, \theta)=\hat{e}(\psi, \theta)=\sum_{k} \psi\left(x_{k}\right) \theta\left(y_{k}\right)=\psi\left(\sum_{k} x_{k} y_{k}\right)=\psi(1)=1 .
$$

Consequently $\hat{y}_{k}(\psi) \neq \hat{y}_{k}(\theta)$ for some $k$ and the claim follows. 
Therefore $M\left(B_{0}\right)$ is homeomorphic to $M(B)$ so the lemma tells us that $\hat{B}_{0}=\hat{B}$, which gives $B=B_{0}+R(B)$. This completes the proof of Theorem 2 .

COROllary. $B$ is separable if and only if $R(B)=R(A) B$ and $\pi$ is a local homeomorphism.

Proof. Assume first that $B$ is separable. $R(B)=R(A) B$ follows from [5, Lemma 1.1, p. 78]. The fact that $\pi$ is a local homeomorphism follows from [10, Theorem 5 , p. 138] or by using [3, Theorem 7.1, (a) $\rightarrow$ (c), p. 72] and our Theorem 1.

For the reverse implication we consider the inertial subalgebra $B_{0}$ of $B$ introduced in the proof of the theorem. Then $M\left(B_{0}\right)=M(B)$. An application of the theorem to $A^{\prime}=A / R(A)$ and to $B^{\prime}=B / R(B)$ (which is a finitely generated projective extension of $A^{\prime}$ because of the equality $\left.R(B)=R(A) B\right)$ leads to the conclusion that $B^{\prime}$ is a strongly separable extension of $A^{\prime}$. For $\phi \in M(A)$, if $m_{\phi}^{\prime}=\left\{a^{\prime} \in A^{\prime} / \phi\left(a^{\prime}\right)=0\right\}$ we obtain natural C-algebra homomorphisms

$$
B_{0} / m_{\phi} B_{0} \stackrel{f}{\rightarrow} B / m_{\phi} B \stackrel{g}{\rightarrow} B^{\prime} / m_{\phi}^{\prime} B^{\prime}
$$

It is easy to see that $g \circ f$ and $g$ are isomorphisms, so $f$ is an isomorphism. Then $\operatorname{dim}_{\mathrm{C}} B / m_{\phi} B=\operatorname{dim}_{\mathrm{C}} B_{0} / m_{\phi} B_{0}$. This means that $B$, which is a finitely generated projective extension of $B_{0}$ (see the proof of the theorem), has (a well-defined) rank 1 over $B_{0}$, that is, $B=B_{0}$. Then $B$ is separable.

4. Proof of Theorem 3. Before giving the proof of the theorem it is convenient to discuss a norm on $B$ which is easily seen to be equivalent to the norm constructed by Magid. Let $\left(b_{j}, u_{j}\right), 1<j<k, b_{j} \in B, u_{j} \in \operatorname{Hom}_{A}(B, A)$, be a dual basis for $B$ over $A\left[3\right.$, p. 4]. If we put $\|b\|^{\prime}=\sum_{j=1}^{k}\left\|u_{j}(b)\right\|, b \in B$, we obtain a norm which induces in $B$ the coarsest topology for which each element of $\operatorname{Hom}_{A}(B, A)$ is continuous. In what follows we shall suppose that each finitely generated projective extension of a Banach algebra is endowed with this Banach algebra topology.

(b) $\rightarrow$ (a). $B_{1}=B \otimes_{A} C(\delta(A))$ is a finitely generated projective extension of $C(\delta(A))$. Using the dual basis $\left(b_{j} \otimes 1, u_{j} \otimes 1\right), 1<j<k$, for $B_{1}$ over $C(\delta(A))$, we see that $B$ is topologically isomorphic to a closed subalgebra of $B_{1}$. Also we have

$$
M\left(B_{1}\right) \simeq \pi^{-1}(\delta(A))=\delta(B) .
$$

If (b) is true, then $B_{1}$ is $C(\delta(A)$ )-separable (use [3, Theorem 7.1, (c) $\rightarrow$ (a), p. 72]). By [2, Theorem 2, p. 30] $B_{1}$ is isomorphic, via the Gelfand transform, to $C(\delta(B))$. But by the open mapping theorem this isomorphism is also topologic. Then $B$ is (topologically isomorphic to) a closed separating subalgebra of $C(\delta(B)$ ) containing the constant functions, that is, $B$ is a uniform algebra.

(a) $\rightarrow$ (b). If $\psi_{1} \in M(B), \phi=\pi\left(\psi_{1}\right)$ and $m\left(\psi_{1}\right)>1$, there exist $b \in B$ such that $\psi_{i}(b)=0$ for each $\psi_{i} \in \pi^{-1}(\phi)$ and $b \notin m_{\phi} B$. This last relation implies that not all $\phi\left(u_{j}(b)\right)$ are 0 , and so we may assume that $\left|\phi\left(u_{1}(b)\right)\right|=2 \delta>0$. For each $\varepsilon>0$ there are neighborhoods $V_{i}$ of $\psi_{i}, 1<i<\operatorname{card} \pi^{-1}(\phi)$, and a neighborhood $U$ of $\phi$, such that $\pi^{-1}(U)=\cup_{i} V_{i},|\psi(b)|<\varepsilon$ for each $\psi \in \pi^{-1}(U)$, and $\left|\omega\left(u_{1}(b)\right)\right|>\delta$ for each $\omega \in U$.

Suppose $\psi_{1} \in \delta(B)$. Then $\phi \in \delta(A)$ and therefore we can find $a \in A$ with 
$\|a\|_{\infty}=1$ and $|a(\omega)|<\min \left(\varepsilon /\|b\|_{\infty}, 1\right)$, for each $\omega \notin U$. According to the hypothesis there exists an $M>0$ such that $\|c\|_{\infty}>M\left(\Sigma_{j}\left\|u_{j}(c)\right\|_{\infty}\right), c \in B$; then

$$
\varepsilon>\|a b\|_{\infty}>M\left(\sum_{j}\left\|u_{j}(a b)\right\|_{\infty}\right)>M\left\|a u_{1}(b)\right\|_{\infty}>M \delta
$$

which is a contradiction because $\varepsilon$ is arbitrary. Thus $\psi_{1} \notin \delta(B)$ and the theorem is proved.

\section{REFERENCES}

1. N. Bourbaki, Commutative algebra, Addison-Wesley, Reading, Mass., 1972.

2. L. N. Childs, On covering spaces and Galois extensions, Pacific J. Math. 37 (1971), 29-33.

3. F. R. DeMeyer and E. C. Ingraham, Separable algebras over commutative rings, Lecture Notes in Math., vol. 181, Springer-Verlag, Berlin, 1971.

4. G. A. Heuer and J. A. Lindberg, Algebraic extensions of continuous function algebras, Proc. Amer. Math. Soc. 14 (1963), 337-342.

5. E. C. Ingraham, Inertial subalgebras of algebras over commutative rings, Trans. Amer. Math. Soc. 124 (1966), 77-93.

6. ___ Inertial subalgebras of complete algebras, J. Algebra 15 (1970), 1-11.

7. J. A. Lindberg, Factorization of polynomials over Banach algebras, Trans. Amer. Math. Soc. 112 (1964), 356-368.

8. Algebraic extensions of commutative Banach algebras, Pacific J. Math. 14 (1964), 559-584.

9. __ Integral extensions of commutative Banach algebras, Canad. J. Math. 25 (1973), 673-686.

10. A. Magid, Algebraically separable extensions of Banach algebras, Michigan Math. J. 21 (1974), 137-143.

11. __, Galois groupoids, J. Algebra 18 (1971), 89-102.

Department de Teoria de Funcions (Matematiques), Universitat de Barcelona, Gran Via No. 585, Barcelona 7, Spain 